\title{
Estrogen receptor $\alpha$ induces down-regulation of PTEN through PI3-kinase activation in breast cancer cells
}

\author{
EUN-MI NOH ${ }^{1 *}$, YOUNG-RAE LEE $^{1 *}$, KEE-OH CHAY ${ }^{4}$, EUN-YONG CHUNG ${ }^{3}$, \\ SUNG HOO JUNG ${ }^{2}$, JONG-SUK KIM ${ }^{1}$ and HYUN JO YOUN ${ }^{2}$ \\ ${ }^{1}$ Department of Biochemistry, Institute for Medical Sciences, Chonbuk National University Medical School, Jeonju 561-180; \\ ${ }^{2}$ Department of Surgery, Chonbuk National University Medical School, Jeonju 560-180; \\ ${ }^{3}$ Department of Anesthesiology and Pain, Catholic University Medical College, Holly Family Hospital, Pucheon 420-818; \\ ${ }^{4}$ Department of Biochemistry, Chonnam National University Medical School, Kwangju 501-746, Republic of Korea
}

Received September 17, 2010; Accepted December 23, 2010

DOI: $10.3892 / \mathrm{mmr} .2011 .412$

\begin{abstract}
Estrogen receptor $\alpha\left(\mathrm{ER}_{\sigma}\right)$ mediates most of the biological effects of estrogen in mammary epithelial cells and stimulates growth signals involving phosphoinositide-3-OH kinase (PI3K)/Akt in breast cancer cells. Phosphatase and tensin homologue (PTEN) is a critical counter-regulator of PI3K signaling and is thus one of the major tumor suppressors in breast cancer. Inhibition of PI3K with an inhibitor, wortmannin, increased the level of PTEN protein in $\mathrm{ER}_{\alpha}$-positive MCF-7 cells, while levels in $\mathrm{ER}_{\alpha}$-negative MDA-MB 231 cells were not altered. In addition, the level of PTEN protein in MCF-7 cells was significantly lower than that in MDA-MB 231 cells, which correlated with high levels of phospho-Akt and phosphatidylinositol-3,4,5,-trisphosphate ( $\mathrm{PIP}_{3}$ ). However, PTEN mRNA expression as measured by real-time PCR showed no differences in either cell line. Notably, the levels of casein kinase 2 (CK2) and phospho-PTEN (Ser380/ Thr382/383) in MCF-7 cells were lower than those in MDA-MB 231 cells, indicating that the down-regulation of PTEN protein in MCF-7 cells is caused by low levels of CK2 expression, leading to accelerated PTEN degradation. Collectively, these results suggest that $\mathrm{ER}_{\alpha}$ induces the down-regulation of PTEN through PI3K activation in breast cancer cells.
\end{abstract}

Correspondence to: Dr Jong-Suk Kim, Department of Biochemistry, Institute for Medical Sciences, Chonbuk National University Medical School, Jeonju 561-180, Korea

E-mail: jsukim@jbnu.ac.kr

Dr Hyun Jo Youn, Department of Surgery, Chonbuk National University Medical School, Jeonju 560-180, Korea

E-mail: yhj0903@jbnu.ac.kr

*Contributed equally

Key words: phosphatase and tensin homologue, protein kinase CK2 or casein kinase II, estrogen receptor, phosphoinositide-3-OH kinase, phosphatidylinositol-3,4,5-trisphosphate

\section{Introduction}

Phosphatase and tensin homologue (PTEN) on chromosome 10q23 is phosphatidylinositol-3' phosphatase (lipid phosphatase) that dephosphorylates the D3 position of phosphatidylinositol3,4,5-trisphosphate ( $\mathrm{PIP}_{3}$ ), a second messenger that is produced by phosphatidylinosititol-3-OH kinase ( $\mathrm{PI} 3 \mathrm{~K})$ and activates protein kinase $\mathrm{B}$ (PKB)/Akt (1-3).

PTEN is known to be one of the major tumor suppressor genes in human cancer and plays a pivotal role in the carcinogenesis of breast cancer cells. PTEN inhibits breast cancer growth through the down-regulation of PI3K signaling, resulting in the blockage of cell cycle progression and the induction of cell death (4). Along the same lines, PTEN has been shown to arrest cells in the $G_{1}$ phase of the cell cycle in breast cancer cell lines (5). These observations suggest that PTEN is involved in the modulation of growth and survival in breast cancer cells. Mutations and/or deletions of PTEN are found in a variety of cancer cells, including endometrial and breast neoplasm cells (4,6-12). In addition, reduced expression of PTEN protein has been observed in approximately one third of all breast cancer cases (13). Moreover, the reduced expression of PTEN protein correlates with lymph node metastasis and a worse prognosis in patients with breast cancer (13-15). However, the down-regulating mechanism of PTEN in breast cancer cells remains to be clarified.

Estrogen plays critical roles in the proliferation of breast cancer cells via two estrogen receptors, estrogen receptor $(\mathrm{ER})_{\alpha}$ and $\mathrm{ER}_{\beta}$, which are ligand-dependent transcription factors that belong to the superfamily of nuclear receptors. In breast cancer, the expression of the ERs is correlated with well-differentiated of tumors and indicates a favorable prognosis, with responsiveness to endocrine therapy with anti-estrogen drugs $(16,17)$. The transcription activities of the ERs are regulated by interacting proteins, such as coactivators and kinases as well as ligand-binding proteins (18). $\mathrm{ER}_{\alpha}$ binds to the p85 regulatory subunit of PI3K in a ligand-dependent manner, thereby activating Akt and subsequently its downstream effectors (19). We have also demonstrated that $\mathrm{ER}_{\alpha}$ stimulates growth signals involving PI3K/Akt in breast cancer 
cells expressing both $\mathrm{ER}_{\alpha}$ and $\mathrm{ER}_{\beta}$ (15). These findings indicate that a PI3K-involved signaling system may be related to the biology of $\mathrm{ER}_{\alpha}$ in breast cancer cells.

One of the traits of cancer cells is an imbalance in signal regulation, and $\mathrm{ER}_{\alpha}$ mediates most of the biological effects of estrogen in mammary epithelial cells. To elucidate the role of $\mathrm{ER}_{\alpha}$ and $\mathrm{ER}_{\beta}$ in the growth of cancer cells, we investigated the regulation of the PI3K signaling system involving PTEN, a counter-regulator of PI3K signaling, in breast cancer cells. Two types of human breast epithelial cell lines were utilized: MCF-7 cells, expressing both $\mathrm{ER}_{\alpha}$ and $\mathrm{ER}_{\beta}$, and MDA-MB-231 cells, expressing $\mathrm{ER}_{\beta}$ only.

\section{Materials and methods}

Materials. Antibodies against PTEN and casein kinase 2 (CK2) were purchased from Santa Cruz Biotechnology (Santa Cruz, CA, USA). Antibodies against phospho-Akt (p-Akt) and phospho-PTEN (p-PTEN) (Ser380/Thr382/383) were from Cell Signaling Technology (Beverly, MD, USA). Fetal bovine serum (FBS), phosphate-buffered saline (PBS) and antibiotics were obtained from Gibco BRL (Life Technologies, Grand Island, NY, USA). Hanks balanced salt solution (HBSS), Dulbecco's modified Eagle's medium (DMEM) and $\beta$-actin antibody were from Sigma Chemical Co. (St. Louis, MO, USA).

Cell culture. The human breast cancer cell lines MCF-7 and MDA-MD-231 were obtained from the American Type Culture Collection (Rockville, MD, USA). The cells were cultured in DMEM containing 10\% FBS and $1 \%$ antibiotics at $37^{\circ} \mathrm{C}$ in a humidified atmosphere $\left(5 \% \mathrm{CO}_{2}, 95 \%\right.$ air).

Western blot analysis. After washing with PBS and harvesting, cell pellets were lysed with $40 \mu \mathrm{l}$ of ice-cold M-PER $^{\circledR}$ Mammalian Protein Extraction Reagent (Pierce Biotechnology, Rockford, IL, USA). Protein concentrations in the lysate were determined using the Bradford method (20). Samples $(30 \mu \mathrm{g})$ were separated by SDS-PAGE with $10 \%$ acrylamide running and 3\% acrylamide stacking gels, and then transferred to hybond ${ }^{\mathrm{TM}}-\mathrm{PVDF}$ membranes using a Western blot apparatus. The PVDF membranes were blotted with $1 \mu \mathrm{g} / \mathrm{ml}$ of primary antibodies for PTEN, p-PTEN, p-Akt, CK2 and $\beta$-actin. Horseradish peroxidase-conjugated $\mathrm{IgG}$ was used as a secondary antibody. Protein expression levels were then determined by analyzing the signals captured on the PVDF membranes using an image analyzer (Las-1000, Fuji-Film, Japan).

Measurement of $\mathrm{PIP}_{3}$ levels. The intracellular $\mathrm{PIP}_{3}$ level was determined by immunocytochemistry using a monoclonal antibody against $\mathrm{PIP}_{3}$ as described by Niswender (21). Briefly, cells mounted on glass slides were equilibrated in PBS at room temperature and then fixed in $4 \%$ paraformaldehyde at room temperature for 5-10 min. After blocking in a blocking buffer containing 5\% normal goat serum and $2 \%$ bovine serum albumin, samples were incubated with a $\mathrm{PIP}_{3}$ monoclonal antibody (Echelon at a 1:100 dilution) overnight at $4^{\circ} \mathrm{C}$. The negative control for the antibody was an equivalent concentration of non-immune mouse IgM. The immunoreac- tivity of $\mathrm{PIP}_{3}$ was detected with goat anti-mouse IgM-TRITC at a 1:200 dilution by incubation for $1 \mathrm{~h}$ at $4^{\circ} \mathrm{C}$. Samples were mounted in aqueous mounting medium with anti-fading agents. Images were acquired with a confocal laser scanning biological microscope (FV 1000; Olympus, Japan).

Quantitative real-time PCR assay. Total RNA was extracted from the cells using TRIzol reagent (Invitrogen, Carlsbad, CA, USA). The concentration and purity of RNA were determined at 260/280 nm. DNA strands were synthesized from $1 \mu \mathrm{g}$ total RNA using oligo-dT primers and AMV reverse transcriptase (Takara, Japan). The expression of PTEN mRNA was determined by real-time reverse transcription-PCR using the ABI-PRISM 7900 sequence detection system (Applied Biosystems, Foster City, CA, USA) and the SYBR Premix Ex Taq (Takara). The primers for real-time reverse transcription-PCR measurement were PTEN primer (NM 002424.2) and 18SrRNA primer (X03205.1) (SuperArray Bioscience Co., Frederick, MD, USA). To control variations in mRNA concentrations, the results were normalized to a housekeeping gene, 18sRNA. Relative quantitation was performed using a comparative $\Delta \Delta \mathrm{Ct}$ method according to the manufacturer's instructions.

Statistical analysis. Statistical data analysis was performed using ANOVA and Duncan's test. Differences with a p-value $<0.05$ were considered statistically significant.

\section{Results}

PI3K modulates PTEN protein levels in human breast cancer cells. To examine the effect of PI3K on PTEN expression,

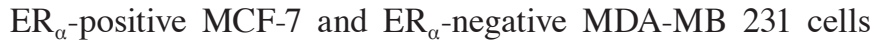
were incubated with 1 and $5 \mu \mathrm{M}$ of wortmannin, a PI3K inhibitor, for $24 \mathrm{~h}$, and the levels of PTEN protein were determined by Western blotting. As shown in Fig. 1, treatment of MCF-7 cells with the inhibitors resulted in a dose-dependent increase in the levels of PTEN protein; PTEN levels were increased by approximately $130 \%$ compared to the control cells. Supporting these results, the levels of p-Akt were decreased (Fig. 1A). However, PTEN levels in MDA-MB 231 cells were not affected by treatment with wortmannin (Fig. 1B).

$E R_{a}$ down-regulates PTEN protein levels in human breast cancer cells. We and others have shown that $\mathrm{ER}_{\alpha}$ activates PI3K in breast cancer cells $(15,19)$. The above results indicate that PI3K modulates PTEN expression. Therefore, PTEN expression profiles and p-Akt levels in $\mathrm{ER}_{\alpha}$-positive MCF-7 cells and $\mathrm{ER}_{\alpha}$-negative MDA-MB 231 cells were determined by Western blotting. PTEN protein levels in $\mathrm{ER}_{\alpha}$-positive MCF-7 cells were significantly lower compared to those in $\mathrm{ER}_{\alpha}$-negative MDA-MB 231 cells, whereas p-Akt levels in MCF-7 cells were higher than those in MDA-MB 231 cells (Fig. 2). To verify these observations, $\mathrm{PIP}_{3}$ levels were also measured using immunocytochemistry. As shown in Fig. 3, $\mathrm{PIP}_{3}$ levels in $\mathrm{ER}_{\alpha}$-positive MCF-7 cells were approximately 20 -fold higher than those in $\mathrm{ER}_{\alpha}$-negative MDA-MB 231 cells.

$E R_{a}$ destabilizes PTEN protein by dephosphorylation in human breast cancer cells. Phosphorylation of PTEN by CK2 
A
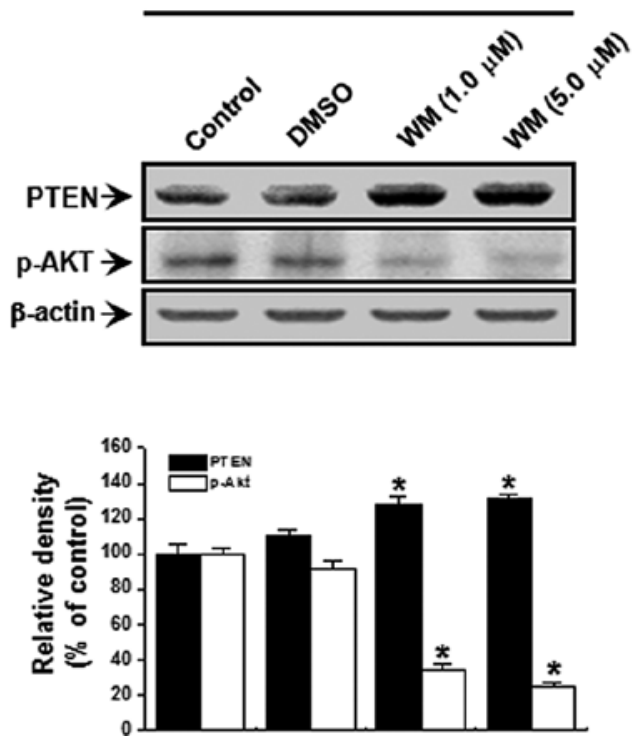

B

MDA-MB 231
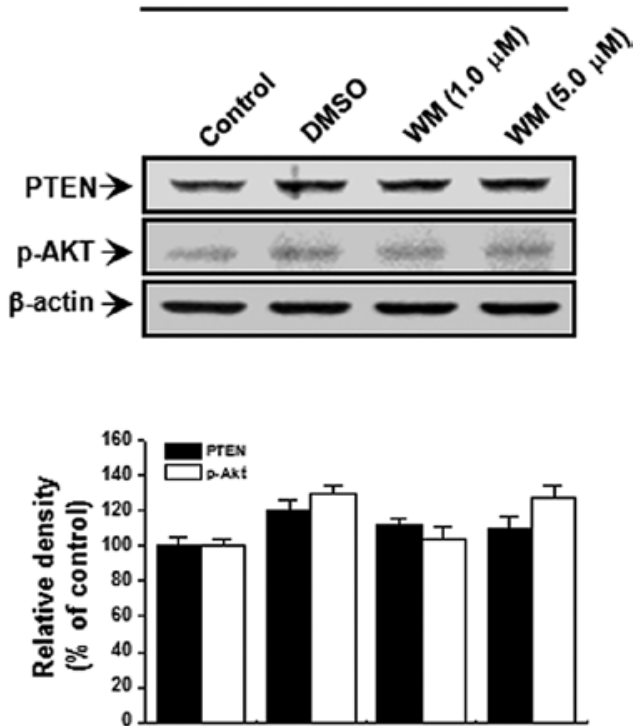

Figure 1. Effect of PI3-Kinase on PTEN expression in breast cancer cells. (A) MCF-7 and (B) MDA-MB 231 human breast cancer cells (2x105) were treated with 1 and $5 \mu \mathrm{M}$ wortmannin for $24 \mathrm{~h}$. Upper panel, cell lysates analyzed by Western blotting for PTEN and p-Akt. $\beta$-actin was used as a loading control. Lower panel, values expressed as a percentage of the control. Error bars, SE; $\mathrm{n}=4$ in each group. " $\mathrm{P}<0.05$ vs. the control.

A

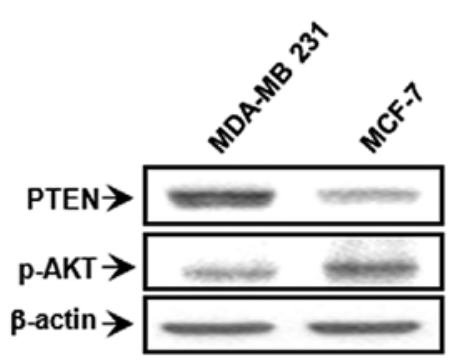

B

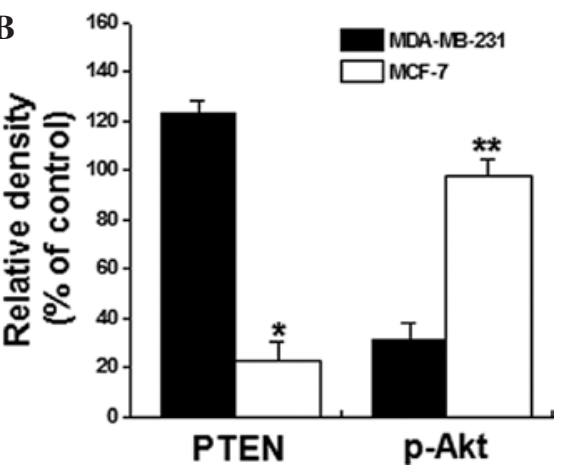

Figure 2. Effect of $\mathrm{ER}_{\alpha}$ on PTEN and p-Akt expression in breast cancer cells. MCF-7 and MDA-MB 231 human breast cancer cells (2x10 $)$ were lysed for Western blotting. (A) Western blot analysis for PTEN and p-Akt. $\beta$-actin was used as a loading control. (B) Values expressed as a percentage of $\beta$-actin. Error bars, $\mathrm{SE} ; \mathrm{n}=4$ in each group. ${ }^{*} \mathrm{P}<0.05,{ }^{* *} \mathrm{P}<0.01$ vs. MDA-MB 231 cells.

has been shown to modulate the stability of PTEN protein (22). To elucidate the mechanism of PTEN down-regulation in ER $_{\alpha}$-positive MCF-7 cells, the levels of PTEN mRNA, p-PTEN and CK2 were determined. There were no differences in the expression of PTEN mRNA between ER $_{\alpha}$-positive

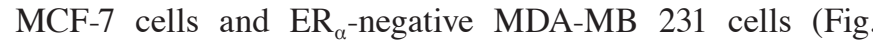
4A). However, the levels of p-PTEN (Ser380/Thr382/383) in MCF-7 cells were significantly lower than those in MDA-MB 231 cells (Fig. 4B). In addition, the levels of CK2 in MCF-7 cells were significantly lower than those in MDA-MB 231 cells (Fig. 4C). These results suggest that the down-regulation of PTEN protein in $\mathrm{ER}_{\alpha}$-positive breast cancer cells is caused by low levels of CK2 expression, leading to a decrease in the phosphorylation of PTEN.

\section{Discussion}

$\mathrm{ER}_{\alpha}$ mediates most of the biological effects of estrogen in mammary epithelial cells and stimulates growth signals involving PI3K/Akt in breast cancer cells (15). To further elucidate the roles of the ERs in breast cancer traits, the regulation of the PI3K signaling system involving PTEN, was evaluated in MCF-7 cells expressing ER $E_{\alpha}$ and ER $E_{\beta}$ and in MDA-MB-231 cells expressing ER $\mathrm{ER}_{\beta}$ only. The results revealed that the levels of PTEN protein in $\mathrm{ER}_{\alpha}$-positive MCF-7 cells were significantly lower than those in $\mathrm{ER}_{\alpha}$-negative MDA-MB 231 cells. Moreover, p-Akt levels in MCF-7 cells were higher than those in MDA-MB 231 cells. In addition, treatment with a PI3K inhibitor, wortmannin, increased the levels of PTEN protein in $\mathrm{ER}_{\alpha}$-positive MCF-7 cells, but not in $\mathrm{ER}_{\alpha}$-negative MDA-MB 231 cells. These results suggest that PI3K signaling reduces the levels of PTEN protein in breast cancer cells expressing $\mathrm{ER}_{\alpha}$, and that the expression of $\mathrm{ER}_{\alpha}$ may differentiate the traits of breast cancer cells.

Deletion or reduced expression of PTEN is associated with poor prognosis, resistance to conventional therapeutic agents and relapse following initial treatment of breast cancer (23-25). PTEN dephosphorylates the D3 position of $\mathrm{PIP}_{3}$, the 
A

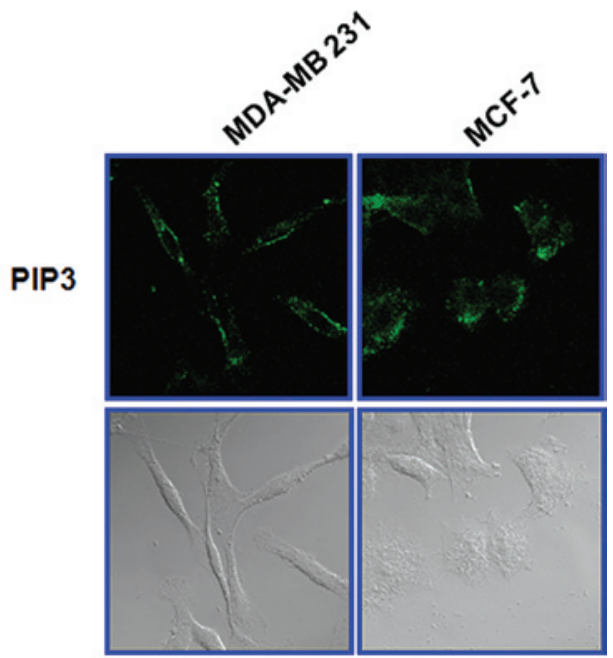

B

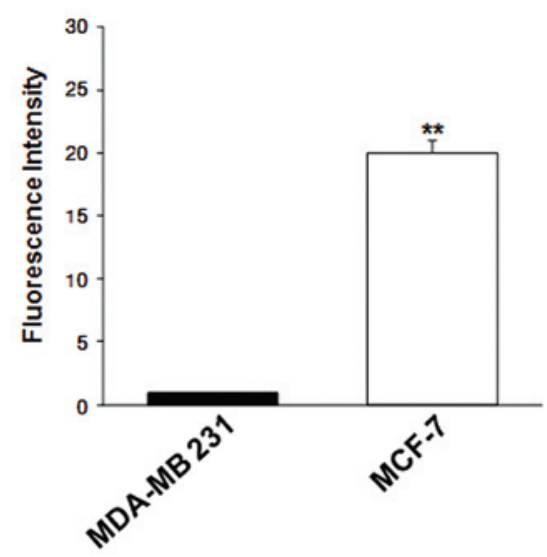

Figure 3. Effect of $\mathrm{ER}_{\alpha}$ on $\mathrm{PIP}_{3}$ in breast cancer cells by immunocytochemistry. To determine $\mathrm{PIP}_{3}$ levels, human breast cancer cells $\left(2 \times 10^{5}\right)$ were fixed with $4 \%$ paraformaldehyde, incubated with anti-PIP ${ }_{3}$ antibody, stained with TRITClabeled anti-mouse $\operatorname{IgM}$ antibody and visualized with confocal fluorescence microscopy. Images were obtained by overlaying fluorescent images and differential interference contrast images. (B) Values are expressed as the mean \pm SE fold increases of cellular $\mathrm{PIP}_{3}(\mathrm{n}=4) .{ }^{* *} \mathrm{P}<0.01$ vs. MDA-MB 231 cells.

second messenger produced by the activation of PI3K (3). Thus, $\mathrm{PIP}_{3}$ levels are controlled directly by the balance of activity between PI3K and PTEN. The results of this study showed that the levels of PTEN protein in $\mathrm{ER}_{\alpha}$-positive MCF-7 cells were lower than those in MDA-MB-231 cells expressing $\mathrm{ER}_{\beta}$ only. Supporting these observations, the levels of $\mathrm{p}-\mathrm{Akt}$ and $\mathrm{PIP}_{3}$ in $\mathrm{ER}_{\alpha}$-positive MCF-7 cells were higher than those in MDA-MB-231 cells.

In this study, we found that a decrease in PTEN levels in $\mathrm{ER}_{\alpha}$-positive MCF-7 cells was due to the degradation of the protein, but not its synthesis. Thus, the levels of PTEN mRNA were similar to each other in both $\mathrm{ER}_{\alpha}$-positive MCF-7 cells and $\mathrm{ER}_{\alpha}$-negative MDA-MB-231 cells. However, the levels

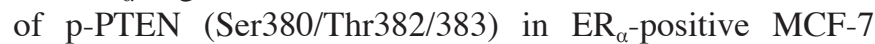
cells were significantly lower than those in $\mathrm{ER}_{\alpha}$-negative MDA-MB 231 cells. Structurally, PTEN is composed of an $\mathrm{N}$-terminal dual specificity phosphatase-like enzyme domain and a C-terminal regulatory domain, which binds to the phospholipid membrane (26). The C-terminal PTEN domain is rich in putative phosphorylation sites, and phosphorylation of the PTEN C-terminus has been reported to affect PTEN stability and function (27).
A
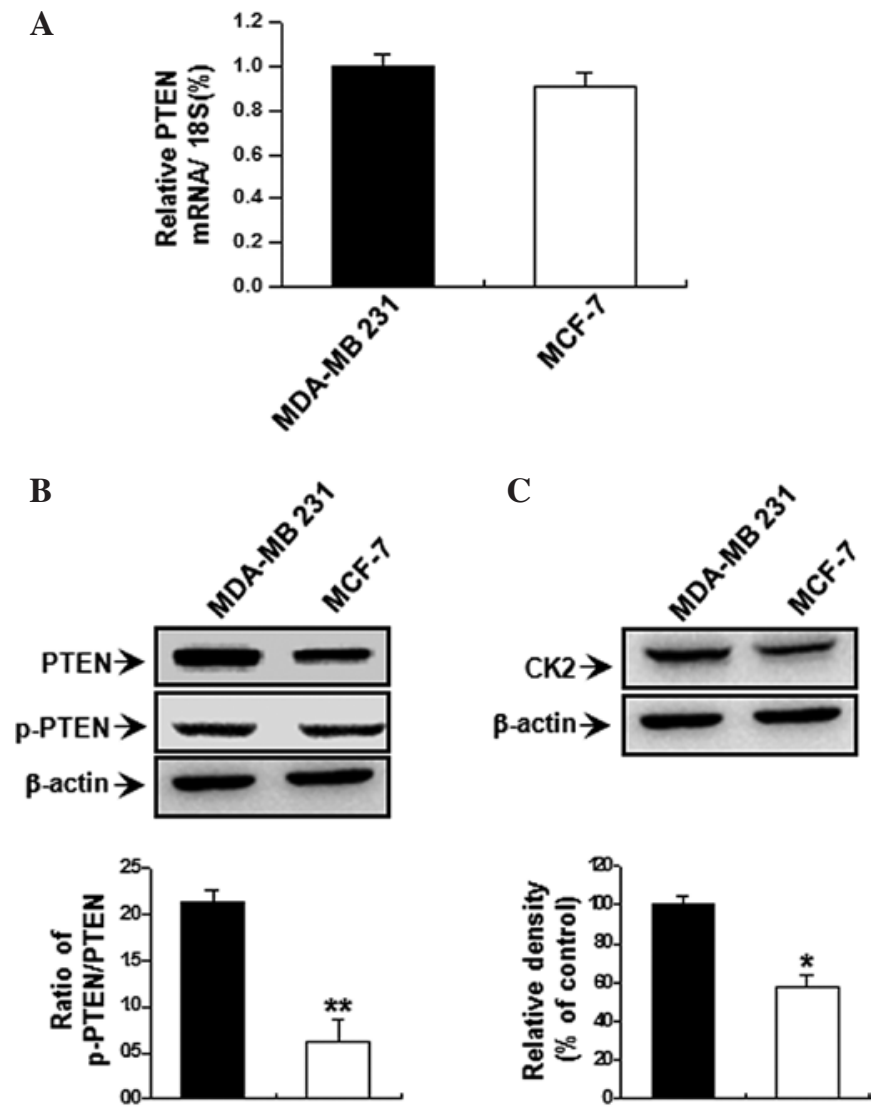

Figure 4. Effect of $\mathrm{ER}_{\alpha}$ on PTEN mRNA, CK2 and p-PTEN expression in breast cancer cells. Human breast cancer cells $\left(2 \times 10^{5}\right)$ were cultured for realtime PCR (PTEN mRNA) and Western blotting (p-PTEN and CK2). (A) PTEN mRNA expression analyzed by real-time PCR. The cell lysates were analyzed by Western blotting for p-PTEN (B) and CK2 (C). Real-time PCR for PTEN was performed as described in Materials and methods. Error bars, $\mathrm{SE} ; \mathrm{n}=4$ in each group. ${ }^{*} \mathrm{P}<0.05,{ }^{* *} \mathrm{P}<0.01$ vs. MDA-MB 231 cells.

Protein kinase CK2 is a messenger-independent serine/ threonine-kinase that phosphorylates a wide variety of substrates involved in essential cell processes, including cell cycle progression and growth $(28,29)$. CK2 is the major kinase involved in the phosphorylation of PTEN, and CK2 phosphorylation sites in PTEN are located within a C-terminal cluster of Ser/Thr residues (30). In addition, inhibition of PTEN phosphorylation with a CK2 inhibitor reduces PTEN protein content, suggesting that proper phosphorylation of PTEN by CK2 is important for the stability of PTEN protein in proteasome-mediated degradation (22). In the present study, we found that the levels of CK2 in MCF-7 cells were significantly lower than those in MDA-MB 231 cells. Taken together, these observations suggest that the down-regulation of PTEN protein in $\mathrm{ER}_{\alpha}$-positive breast cancer cells is caused by low levels of CK2 expression, leading to accelerated PTEN degradation.

In conclusion, our results indicate that PTEN is down-regulated in $\mathrm{ER}_{\alpha}$-positive, but not in $\mathrm{ER}_{\alpha}$-negative, breast cancer cells, and that the down-regulation of PTEN is caused by the reduced phosphorylation of PTEN. The results also suggest that PTEN expression is regulated through PI3K signaling involving $\mathrm{ER}_{\alpha}$ in breast cancer cells. Since deletion or reduced expression of PTEN is known to be associated with poor outcome in breast cancer, our findings may aid in the design 
of novel therapeutic strategies as well as in the diagnosis of $\mathrm{ER}_{\alpha}$-positive breast cancer.

\section{Acknowledgements}

The authors would like to thank Professor Mie-Jae Im for the critical review of the manuscript. This study was supported by a research fund from the Chonbuk National University Hospital Research Institute of Clinical Medicine, and by a grand from the National Research Foundation of Korea (NRF) funded by the Korea Government (MEST) (no. 2010-0001355, no. 2010-0012716 and M10528010003-05N2801-00310).

\section{References}

1. Tonks NK and Myers MP: Structural assets of a tumor suppressor. Science 286: 2096-2097, 1999.

2. Myers MP, Pass I, Batty IH, et al: The lipid phosphatase activity of PTEN is critical for its tumor supressor function. Proc Natl Acad Sci USA 95: 13513-13518, 1998.

3. Maehama T and Dixon JE: The tumor suppressor,PTEN/MMAC1, dephosphorylates the lipid second messenger, phosphatidylinositol 3,4,5-trisphosphate. J Biol Chem 273: 13375-13378, 1998.

4. Weng LP, Smith WM, Dahia PL, et al: PTEN suppresses breast cancer cell growth by phosphatase activity-dependent G1 arrest followed by cell death. Cancer Res 59: 5808-5814, 1999.

5. Weng LP, Brown JL and Eng C: PTEN coordinates G1 arrest by down-regulating cyclin D1 via its protein phosphatase acitivity and up-regulating p27 via its lipid phosphatase acitivity in a breast cancer model. Hum Mol Genet 10: 599-604, 2001.

6. Eng C, Strallon M, Ponder B, et al: Familial cancer syndromes. Lancet 343: 709-713, 1994

7. Liaw D, Marsh DJ, Li J, et al: Germline mutations of the PTEN gene in Cowden disease, an inherited breast and thyroid cancer syndrome. Nat Genet 16: 64-67, 1997.

8. Eng $\mathrm{C}$ and Peacocke M: PTEN and inherited hamartoma-cancer syndromes. Nat Genet 19: 223, 1998.

9. Ali IU, Schriml LM and Dean M: Mutational spectra of PTEN/ MMAC1 gene: a tumor suppressor with lipid phosphatase activity. J Natl Cancer Inst 91: 1922-1932, 1999.

10. Garcia JM, Silva JM, Dominguez G, et al: Allelic loss of the PTEN region (10q23) in breast carcinomas of poor pathophenotype. Breast Cancer Res Treat 57: 237-243, 1999.

11. Perren A, Weng LP, Boag AH, et al: Immunohistochemical evidence of loss of PTEN expression in primary ductal adenocarcinomas of the breast. Am J Pathol 155: 1253-1260, 1999.

12. Vivanco I and Sawyers CL: The phosphatidylinositol 3-Kinase AKT pathway in human cancer. Natl Rev Cancer 2: 489-501, 2002.

13. Tsutsui S, Inoue H, Yasuda K, Suzuki K, Higashi H, Era S and Mori M: Reduced expression of PTEN protein and its prognostic implications in invasive ductal carcinoma of the breast. Oncology 68: 398-404, 2005.

14. Depowski PL, Rosenthal SI and Ross JS: Loss of expression of the PTEN gene protein product is associated with poor outcome in breast cancer. Mod Pathol 14: 672-676, 2001.
15. Lee YR, Park J, Yu HN, Kim JS, Youn JH and Jung SH: Up-regulation of PI3K/Akt signaling by 17beta-estradiol through activation of estrogen receptor-alpha, but not estrogen receptorbeta, and stimulates cell growth in breast cancer cells. Biochem Biophys Res Commun 336: 1221-1226, 2005.

16. Clarke R, Skaar T, Baumann K, et al: Hormonal carcinogenesis in breast cancer: cellular and molecular studies of malignant progression. Breast Cancer Res Treat 31: 237-248, 1994.

17. Ali S and Coombes RC: Endocrine-responsive breast cancer and strategies for combating resistance. Nat Rev Cancer 2: 101-112, 2002.

18. Ikeda $\mathrm{K}$ and Inoue $\mathrm{S}$ : Estrogen receptors and their downstream targets in cancer. Arch Histol Cytol 67: 435-442, 2004.

19. Simoncini T, Hafezi-Moghadam A, Brazil DP, Ley K, Chin WW and Liao JK: Interaction of oestrogen receptor with the regulatory subunit of phosphatidylinositol-3-OH kinase. Nature 407: 538-541, 2000.

20. Bradford MM: A rapid and sensitive method for the quantitation of microgram quantities of protein utilizing the principle of protein-dye binding. Anal Biochem 72: 248-254, 1976.

21. Niswender KD, Gallis B, Blevins JE, Corson MA, Schwartz MW and Baskin DG: Immunocytochemical detection of phosphatidylinositol 3-kinase activation by insulin and leptin. J Histochem Cytochem 51: 275-283, 2003.

22. Torres J and Pulido R: The tumor suppressor PTEN is phosphorylated by the protein kinase $\mathrm{CK} 2$ at its $\mathrm{C}$ terminus. Implications for PTEN stability to proteasome-mediated degradation. J Biol Chem 276: 993-998, 2001.

23. Perez-Tenorio G, Stål O; Southeast Sweden Breast Cancer Group: Activation of AKT/PKB in breast cancer predicts a worse outcome among endocrine treated patients. Br J Cancer 86: 540-545, 2002.

24. Nagata Y, Lan KH, Zhou X, et al: PTEN activation contributes to tumor inhibition by trastuzumab, and loss of PTEN predicts trastuzumab resistance in patients. Cancer Cell 6: 117-127, 2004.

25. Shoman N, Klassen S, McFadden A, Bickis MG, Torlakovic E and Chibbar R: Reduced PTEN expression predicts relapse in patients with breast carcinoma treated by tamoxifen. Mod Pathol 18: 250-259, 2005.

26. Lee JO, Yang H, Georgescu MM, et al: Crystal structure of the PTEN tumor suppressor: implications for its phosphoinositide phosphatase activity and membrane association. Cell 99: 323-334, 1999

27. Vazquez F, Ramswamy S, Nakamura $\mathrm{N}$ and Sellers WR: Phosphorylation of the PTEN tail regulates protein stability and function. Mol Cell Biol 20: 5010-5018, 2000.

28. Meisner $\mathrm{H}$ and Czech MP: Phosphorylation of transcriptional factors and cell-cycle-dependent proteins by casein kinase II. Curr Opin Cell Biol 3: 474-483, 1991.

29. Allende JE and Allende CC: Protein kinase 4. Protein kinase CK2: an enzyme with multiple substrates and a puzzling regulation FASEB J 9: 313-323, 1995.

30. Al-Khouri AM, Ma Y, Togo SH, Williams S and Mustelin T: Cooperative phosphorylation of the tumor suppressor phosphatase and tensin homologue (PTEN) by casein kinases and glycogen synthase kinase 3beta. J Biol Chem 280: 35195-35202, 2005 . 